\title{
Compliant Constant-Force Linear-Motion Mechanism
}

\author{
Kyler A. Tolman, Ezekiel G. Merriam, Larry L Howell ${ }^{1}$ \\ Department of Mechanical Engineering \\ Brigham Young University \\ Provo, Utah 84602
}

\begin{abstract}
A fully compliant constant-force mechanism that uses an initially angled parallelguiding mechanism is proposed. A pseudo-rigid-body model (PRBM) of the mechanism is developed and validated using both finite element models and experimental prototype testing. The PRBM is used as a preliminary design tool to identify parameters that result in a constant-force mechanism. An adjustable version of the mechanism is also proposed and is used to assess the range of validity of the PRBM and highlights how the system response can be varied by changing the flexible beams inclination. A finite element based optimization is used to create a modified version of the mechanism that incorporates beam-end rotation effects and offers greater performance. Potential applications for the mechanism are discussed and it is demonstrated as a component of a statically balanced system.
\end{abstract}

\section{Introduction}

A constant-force mechanism $(\mathrm{CFM})$ has a constant reaction force over a range of displacements. These mechanisms have potential in applications where a certain amount of contact force is desired such as in electrical connectors [1, 2] 5 and automotive clutches [3, 4]. CFM's can be used in exercise equipment to simulate free weights [5, 6], in robot end-effector applications [7, 8] and grippers for handling objects of various sizes 9 . Adjustable constant-force mechanisms have been achieved by combining bistable mechanisms with preloaded linear springs [10. CFM's have also been combined with bistable mechanisms to 10 provide overload protection in force regulation applications [11. Applications for constant-force mechanisms range from devices such as snap fits [12, to microelectromechanical systems [13, 14].

Early constant-force mechanisms were constant-force tension springs which are also known as Negator springs [15. These springs consist of a strip of 15 spring steel in a tightly wound coil and are found in consumer items such as tape measures and pull-starts. Nathan proposed a rigid link constant-force generator

\footnotetext{
${ }^{1}$ Corresponding author. lhowell@byu.edu
}

Preprint submitted to Journal of Mechanisms and Machine Theory

August 22, 2016

(c) 2016. This manuscript version is made available under the Elsevier user license http://www.elsevier.com/open-access/userlicense/1.0/ 


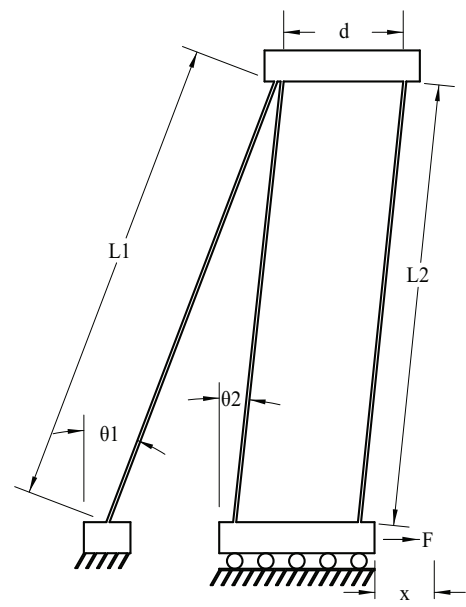

Figure 1: Proposed constant-force mechanism.

which produces a constant unidirectional force for any position [16. This basic mechanism is found in many gravitational force balancing applications such as desk lamps. Other rigid link-mechanisms which produce constant-force have

20 also been developed [17. Methods for the design, analysis, and application of compliant constant-force mechanisms continues to be a topic of research [18, 19, 20, 21, 22, 23.

The compliant constant-force linear-motion mechanism proposed in this paper combines constant-force characteristics with a parallel guiding mechanism.

25 This mechanism consists of an initially tilted parallel guiding mechanism on a sliding joint and a grounded connecting link as shown in Figure 1.

By mirroring this mechanism along the ground link, a slider is no longer needed and the mechanism can achieve constant-force displacement without sliders or revolute joints, i.e. it is a fully compliant constant-force mechanism.

30 This mechanism (Figure 2) has the potential to be used in MEMS devices as a statically balancing mechanism, in electrical connectors, or in any application where constant force is desired.

\section{Analytic Basis for the Mechanism}

The basis of the mechanism is a compliant parallel guiding mechanism that has been fabricated with an initial tilt $\theta_{2}$ as shown in Figure 1. The parallel guiding mechanism is then attached to a slider and a grounded connecting link. A pseudo rigid body model (PRBM) 24, 25] for the mechanism was developed where the flexible segments are modeled as rigid links with torsional springs, as shown in Figure 3. The spring constant $K$ of the PRBM torsional springs is 40 given by

$$
K=2 \gamma K_{\Theta} \frac{E I}{l}
$$




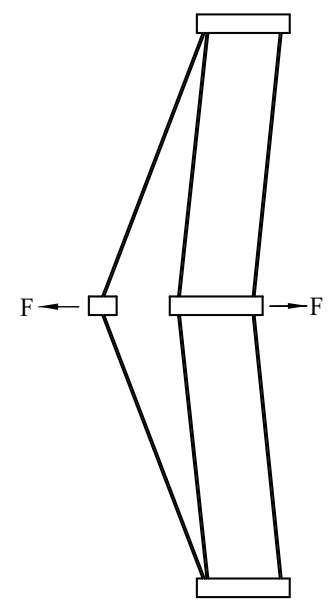

Figure 2: A fully compliant version of the constant-force mechanism.

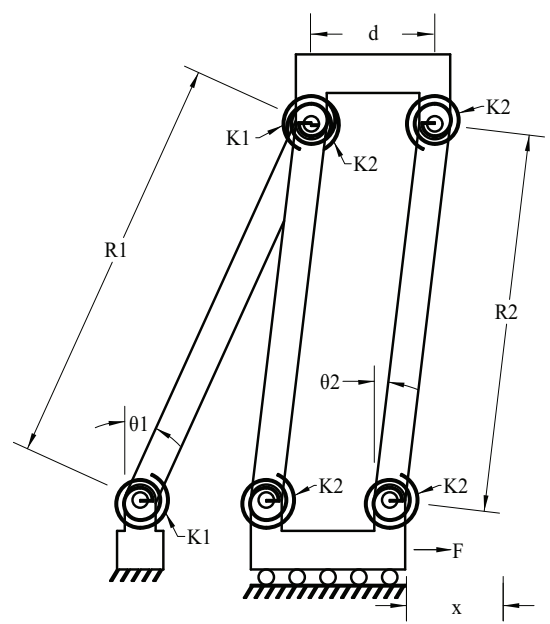

Figure 3: PRBM of the compliant constant-force mechanism.

where $K_{\Theta}=2.5$ and $\gamma=0.8$. These values were found by minimizing the difference in system response predicted by the PRBM, and the finite element model which is discussed in section 4 . The link lengths $R_{1}$ and $R_{2}$ are obtained respectively by

$$
R_{i}=\gamma L_{i}
$$

45 where $L_{i}$ corresponds to the compliant link lengths $L_{1}$ and $L_{2}$. Because the motion of the two legs attached to the parallel guiding link is the same, this model can be simplified into the basic crank slider mechanism as shown in Figure 4. 


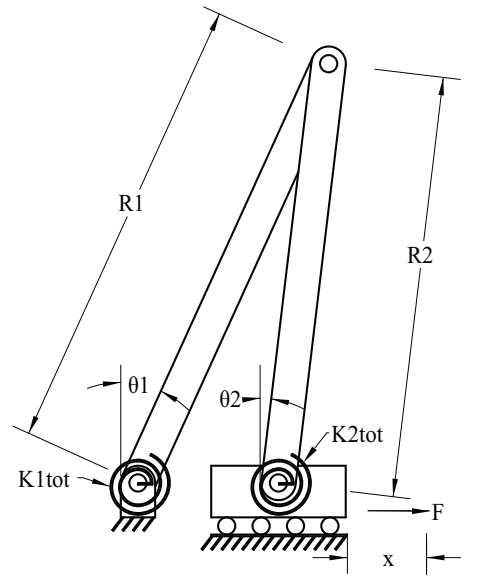

Figure 4: Simplified crank-slider model of compliant parallel-guiding constant-force mechanism.

Using the principle of virtual work 24], an expression for the output force in terms of angular displacement was found as:

$$
\begin{aligned}
F=K_{1_{\text {tot }}}\left(\theta_{1}{ }^{\prime}-\theta_{1}\right)\left(\frac{-\sin \left(\theta_{2}{ }^{\prime}\right)}{R_{1} \sin \left(\theta_{1}{ }^{\prime}-\theta_{2}{ }^{\prime}\right)}\right) & \\
& +K_{2_{\mathrm{tot}}}\left(\theta_{2}{ }^{\prime}-\theta_{2}\right)\left(\frac{-\sin \left(\theta_{1}{ }^{\prime}\right)}{R_{2} \sin \left(\theta_{1}{ }^{\prime}-\theta_{2}{ }^{\prime}\right)}\right)
\end{aligned}
$$

where $K_{1_{\text {tot }}}=2 K_{1}, K_{2_{\text {tot }}}=4 K_{2}, \theta_{1}$ and $\theta_{2}$ are the beam angles in the unde50 flected position, and $\theta_{1}{ }^{\prime}$ and $\theta_{2}{ }^{\prime}$ correspond to the beam angles in the deflected position. Furthermore the linear displacement $(x)$ of this mechanism can be related to the angular displacement of the beams by:

$$
x=\left(R_{1} \sin \left(\theta_{1}{ }^{\prime}\right)-R_{2} \sin \left(\theta_{2}{ }^{\prime}\right)\right)-\left(R_{1} \sin \left(\theta_{1}\right)-R_{2} \sin \left(\theta_{2}\right)\right)
$$

It was discovered through an optimization of parameters, discussed in Section 3. that the bending stiffness of the segment $L_{1}$ should be minimized to achieve greater constant-force displacement. On the macro scale this member could be constructed from a cable or wire. The resulting stiffness of this member would then be negligible in comparison to the other members and the first expression of Equation (3) can be ignored and the expression is then simplified to

$$
F=K_{2_{\mathrm{tot}}}\left(\theta_{2}{ }^{\prime}-\theta_{2}\right)\left(\frac{-\sin \left(\theta_{1}^{\prime}\right)}{R_{2} \sin \left(\theta_{1}^{\prime}-\theta_{2}^{\prime}\right)}\right)
$$




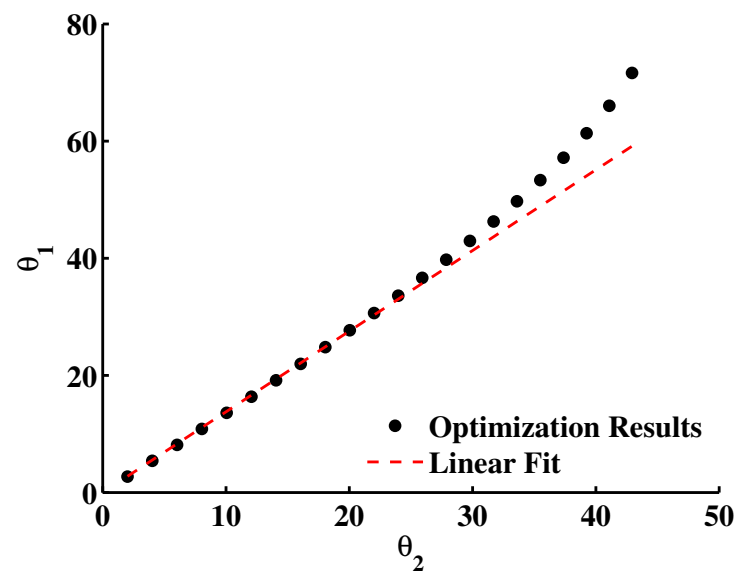

Figure 5: Optimization results of initial angle combinations that result in a constant-force relationship.

\section{Optimization of Parameters}

Using the simplified crank-slider model of the mechanism and the force expression from Equation (3) a generalized reduced gradient nonlinear optimization routine was used to determine the dimensional parameters that would result in an optimized constant-force mechanism. The optimization design variables

65 were the beam angles $\theta_{1}$ and $\theta_{2}$, and the beam thicknesses. Other dimensions such as the beam length $L_{2}$, and beam depth were fixed. The objective function was to minimize the difference between a chosen constant-force value $F_{\text {ideal }}$ and the output force $F$ between displacements $\theta_{2} / 2$ and $-\theta_{2} / 2$, or

$$
\begin{aligned}
\operatorname{minimize}\left|F-F_{\text {ideal }}\right| & \\
& \text { with respect to: } \theta_{1}, \theta_{2}, t_{1}, t_{2} \\
& \text { subject to: } \frac{\theta_{2}}{2}<\theta_{2}{ }^{\prime}<\frac{-\theta_{2}}{2}
\end{aligned}
$$

The optimization found that the ratio of $\theta_{1}$ and $\theta_{2}$ is an important factor in determining force-displacement behaviour. As noted before, it was also found that the stiffness of beam 1 , or beam thickness $t_{1}$ should be reduced to generate a larger constant-force displacement. As shown in Figure 5 for $0^{\circ}<\theta_{2}<35^{\circ}$ the solution of initial angle combinations that result in a CFM is nearly linear. When approximating this data with a linear fit, we find that an initial angle of $75 \theta_{1}=1.35 \theta_{2}$ will produce a mechanism with constant-force behavior. 


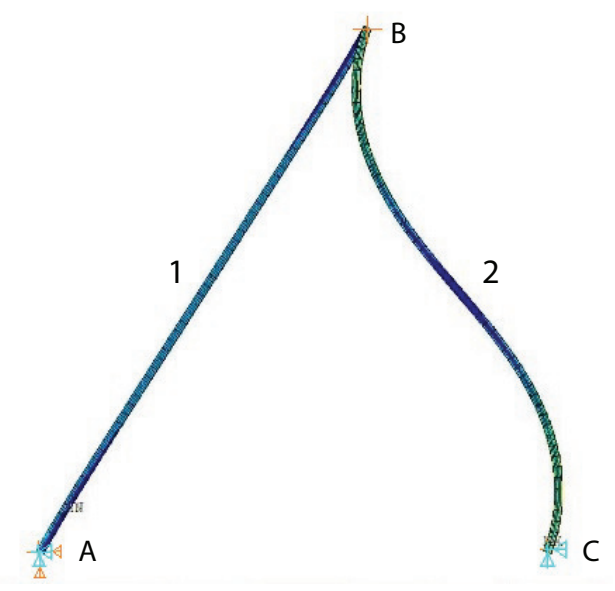

Figure 6: Displaced FEA model of the constant-force mechanism.

\section{Model Validation}

\subsection{Finite Element Analysis}

The compliant constant-force linear-motion mechanism was validated using finite element analysis (FEA). The finite element (FE) model is comprised of two beams meshed together in the position of the crank slider, as shown in Figure 6 . The parameters used in the model can be found in table1. It must be noted that the width of beam 2 was doubled because the FE model utilizes one beam to represent the two flexible members of the parallel guiding portion of the CFM. A nonlinear analysis was performed using ANSYS FEA software with beam188

85 elements. The mechanism was fixed in all directions at the beam end section at point $\mathrm{A}$ and the beam end sections at points $\mathrm{B}$ and $\mathrm{C}$ were fixed in rotation to simulate fixed-guided beam conditions. Point $\mathrm{C}$ was displaced horizontally and the reaction forces were calculated. The FEA results are compared to the PRBM in Figure 9 and reveal that the PRBM is within approximately seven

90 percent of the FEA results on average.

\subsection{Experimental Prototype}

A fully compliant experimental prototype consisting of two halves of the CFM was fabricated using the parameters listed in Table 1. These parameters are also the parameters used in both the PRBM and FE models. Parameters such as the beam thickness $(t)$ and width $(w)$ were chosen based upon what would result in lower stress levels and could be conveniently 3D printed. Other parameters such as beam lengths and angles were based upon the optimized results given by the PRBM. The prototype was fabricated out of polylactic acid (PLA) using a Makerbot ${ }^{\circledR}$ Replicator ${ }^{\mathrm{TM}} 2$ 3D printer. The modulus of elasticity $(E)$ as listed in Table 1 is the modulus specified by the manufacturer of the 3D 


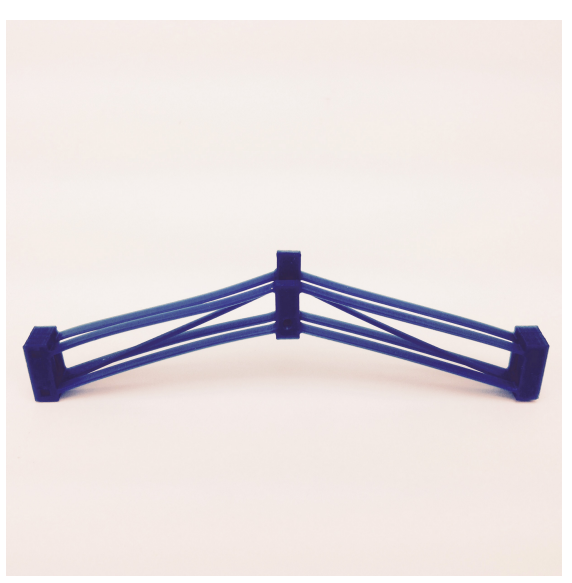

(a) Front view of CFM.

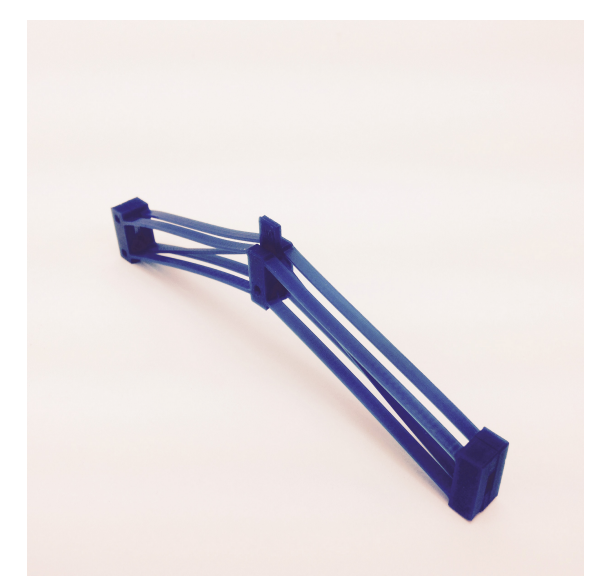

(b) Side view of CFM.

Figure 7: Prototype Constant-Force Mechanism (CFM) 3D printed out of PLA.

printing filament. Due to the 3D printing process the resulting mechanical properties of the prototype can vary due to a non fully dense material created by the fused deposition modeling (FDM) process. The prototype (shown in Figure 7), was fabricated in three parts: two identical parts that made up the 105 parallel guiding mechanism and one piece that made up link $L_{1}$. The prototype was tested using an Instron tensile testing machine as seen in Figure 8.

\subsection{Results}

The experimental results shown in Figure 9 agree with both the PRBM and FEA data in the force-displacement behavior. The force values predicted by the

Table 1: Parameters used for the PRBM, FE, and experimental models.

\begin{tabular}{lcc} 
Parameter & Value & Units \\
\hline \hline$L_{1}$ & 57.2 & $\mathrm{~mm}$ \\
$L_{2}$ & 54 & $\mathrm{~mm}$ \\
$w_{1}$ & 1 & $\mathrm{~mm}$ \\
$w_{2}$ & 6.35 & $\mathrm{~mm}$ \\
$t_{1}$ & 0.8 & $\mathrm{~mm}$ \\
$t_{2}$ & 0.8 & $\mathrm{~mm}$ \\
$\theta_{1}$ & 28.35 & $\mathrm{deg}$ \\
$\theta_{2}$ & 21 & $\mathrm{deg}$ \\
$E$ & $3.31 \times 10^{9}$ & $\mathrm{~Pa}$ \\
$K_{\theta}$ & 2.5 & - \\
$\gamma$ & 0.8 & - \\
$d$ & 15 & $\mathrm{~mm}$ \\
\hline
\end{tabular}




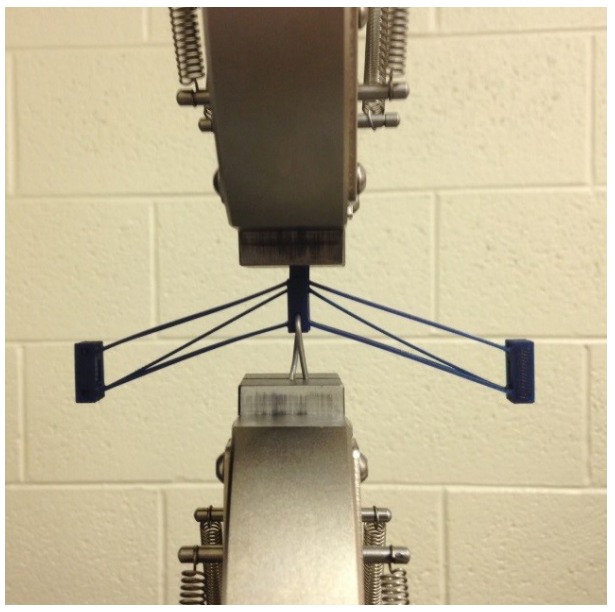

Figure 8: Force-deflection testing of 3D printed prototype.

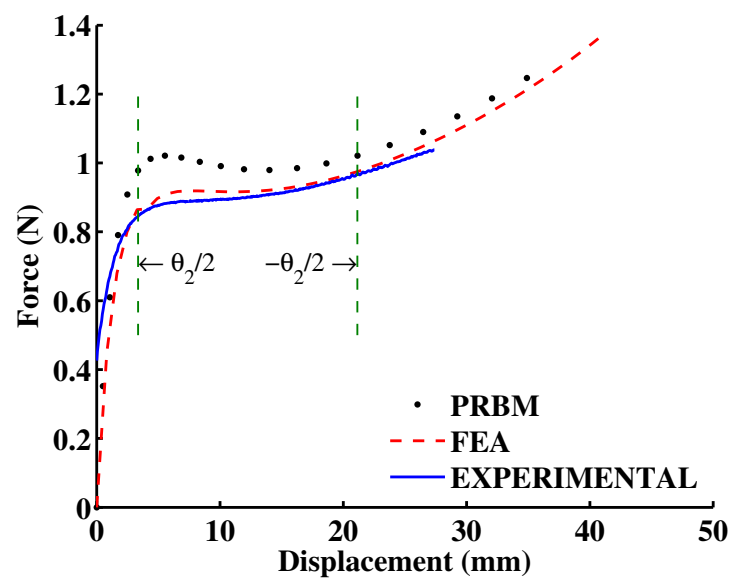

Figure 9: Experimental results of the prototype mechanism. The optimization range as stated in Equation 6 is identified by the vertical dashed lines.

three percent. It must be noted that the experimental result shown is half the force output of the actual prototype, as the prototype consists of two CFM's joined into one, allowing it to be fully compliant. It can be observed in these results that the PRBM predicts a somewhat sinusoidal response whereas this is not as pronounced in the FEA and experimental results. This can be explained by the difference in the models; the pseudo-rigid-body model assumes rigid links connected by revolute pairs with torsional springs in parallel whereas the FEA assumes fully deformable links. 


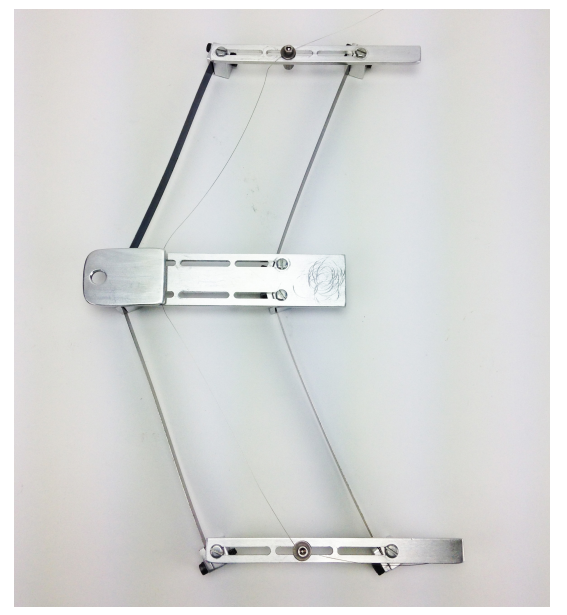

Figure 10: Adjustable constant-force mechanism.

\section{Adjustable Mechanism}

An adjustable constant-force mechanism was designed and tested to validate the beam angle ratio required to produce constant force at different angles. The mechanism (shown in Figure 10 was constructed using thin flexible steel members and rigid aluminum blocks. The fixed parameters of this adjustable mechanism are listed in Table 2. The aluminum blocks can be adjusted so that

125 the un-strained position of the parallel guiding mechanism can range between $-80^{\circ}<\theta_{2}<80^{\circ}$ without interference. The mechanism is actuated by pulling on an aluminum tab which is attached to link $L_{1}$ (see Figure 1) which is fabricated out of a steel wire and can also be adjusted to form different inclinations similar to links $L_{2}$.

130 The adjustable constant-force mechanism was tested using the custom-built force-displacer test device shown in Figure 11. It consists of a linear actuator, a

Table 2: Fixed parameters of the adjustable CFM.

\begin{tabular}{lcr} 
Parameter & Value & Units \\
\hline \hline$L_{2}$ & 138.43 & $\mathrm{~mm}$ \\
$w_{1}$ & 0.216 & $\mathrm{~mm}$ \\
$w_{2}$ & 12.7 & $\mathrm{~mm}$ \\
$t_{1}$ & 0.216 & $\mathrm{~mm}$ \\
$t_{2}$ & 0.305 & $\mathrm{~mm}$ \\
$E$ & $210 \times 10^{9}$ & $\mathrm{~Pa}$ \\
$K_{\theta}$ & 2.5 & - \\
$\gamma$ & 0.8 & - \\
$d$ & 89 & $\mathrm{~mm}$ \\
\hline
\end{tabular}




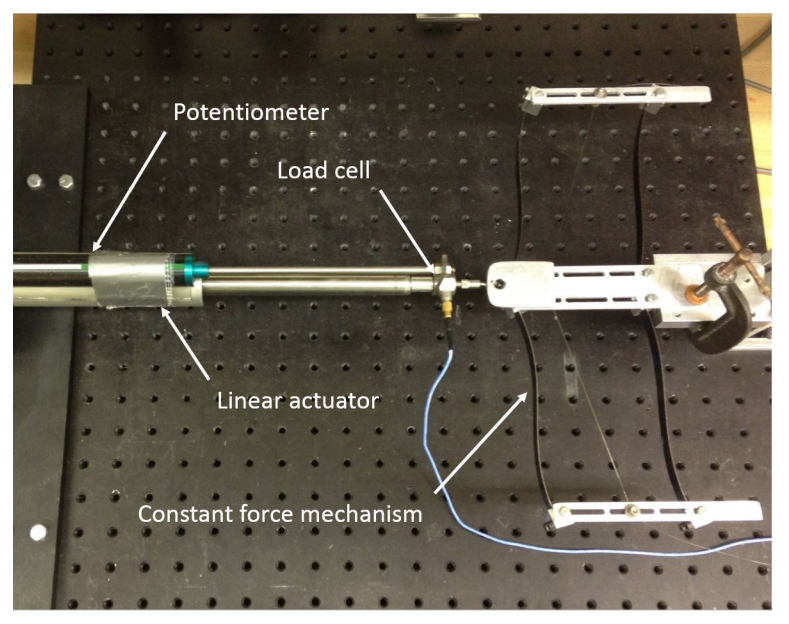

Figure 11: Setup for testing the force-displacement of the adjustable mechanism.

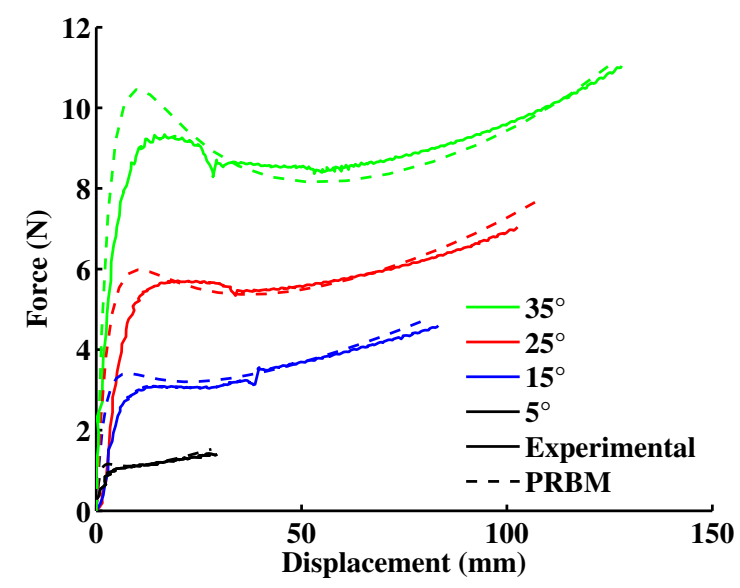

Figure 12: Force-displacement of adjustable mechanism with varying initial angles.

Kistler model 9212 quartz dynamic load cell, and a linear potentiometer to sense position. Initial beam angles $\left(\theta_{2}\right)$ from $0^{\circ}$ to $60^{\circ}$ in increments of $5^{\circ}$ were tested using the setup. The beam angle for $L_{1}$ was adjusted accordingly such that ${ }_{135} \theta_{1}=1.35 \theta_{2}$ in each experiment. The results for four different angles from $5^{\circ}$ to $35^{\circ}$ are shown in Figure 12. The measured force-displacement behavior closely correlates with PRBM predictions. For angles smaller than $5^{\circ}$ the magnitude of constant force and the displacement range were small. For angles greater than $35^{\circ}$, the PRBM diverges from the experimental results as beam buckling effects begin to occur. 


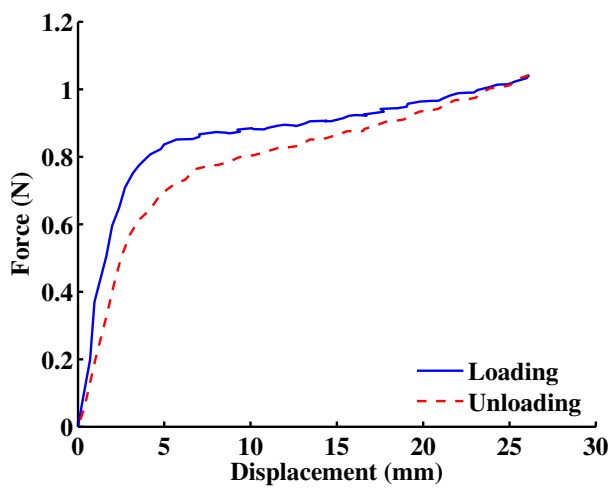

(a) Cyclic loading of PLA CFM

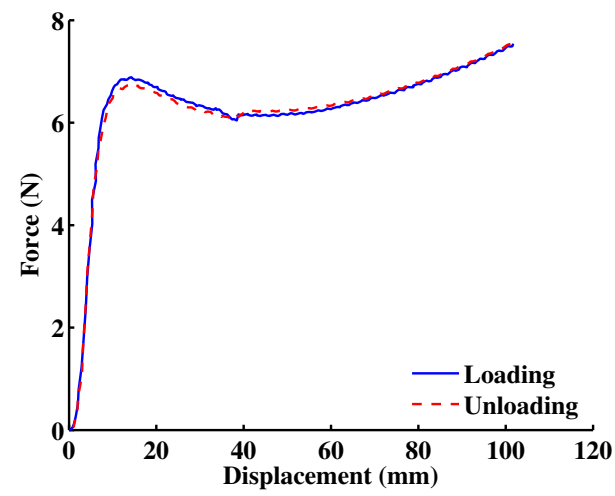

(b) Cyclic loading of steel CFM

Figure 13: Experimental results comparing the loading-unloading behavior of both the PLA and steel constant-force mechanisms.

After examining the linear model fit in Figure 5, we can see that the optimization results diverge from the linear fit at around $\theta_{2}=40^{\circ}$. In this same region the PRBM predictions diverge from the FEA predictions. When designing constant-force mechanisms that use parallel guiding mechanisms that are initially angled greater than $40^{\circ}$, FEA will give more accurate results than the PRBM.

Along with validating the models from $0^{\circ}$ to $35^{\circ}$, this adjustable mechanism also shows the feasibility of designing mechanisms that are able to alter their level of constant-force by changing the beam angles. This design can be implemented in scenarios where different levels of constant-force may be desired of the same mechanism, such as in balancing devices.

\section{Unloading Behavior}

Because the compliant CFM can be used in applications requiring frequent loading and unloading, it is of interest to examine hysteresis effects on the mechanism. Using the same force-displacer test device described in section 5, the adjustable steel CFM and the original PLA prototype CFM were tested in both loading and unloading at an average rate of $4.6 \mathrm{~mm} / \mathrm{s}$. The results are shown in Figure 13. We can see from these experimental results that the unloading force-displacement of the PLA prototype differs from the loading behavior, as expected for a polymer like PLA. The steel prototype, on the other hand experienced little hysteresis. Thus for applications where hysteresis would present a problem, the mechanism should be fabricated from materials that exhibit minimal losses. 


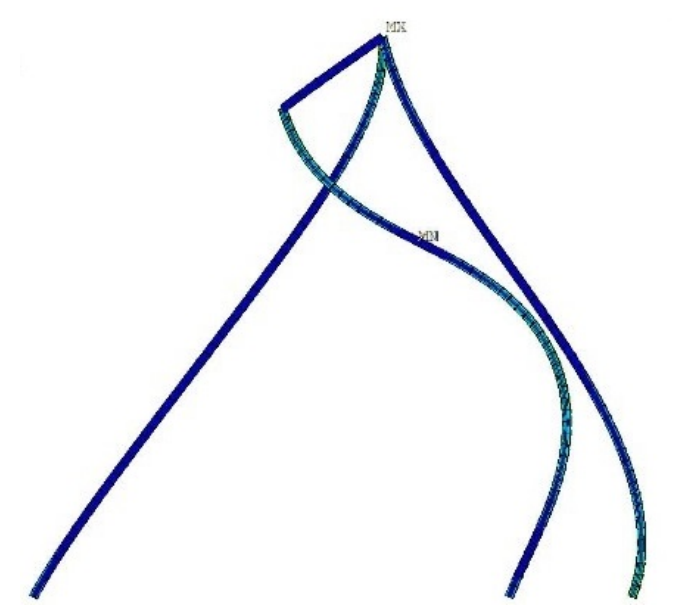

Figure 14: FEA model of the modified design which takes into account beam end rotation.

\section{Modified Design}

The compliant constant-force linear motion-mechanism is modeled based on the assumption that the parallel-guiding link remains parallel in its motion. However the moment placed on this link causes a slight rotation in the compliant version. This effect increases as the flexures are placed closer together. It was observed in early 3D printed prototypes where the legs were too close together 170 that this phenomenon could aid the mechanism in achieving a greater constantforce stroke.

To explore the effects of a twisting parallel-guiding link more fully, a FE model consisting of the entire half of the mechanism was constructed. The FE model in Figure 14 was run through a Matlab fminunc optimization routine where the spacing between flexible members and the beam angles were used as design variables. Other dimensions were fixed and the objective of the optimization was to minimize the difference in resulting forces between displacements of $\theta_{2} / 2$ and $-\theta_{2}$. This is similar to the criteria used in the optimization of the original design, but with final displacement being $-\theta_{2}$ rather than $-\theta_{2} / 2$. For

180 this prototype parameters such as the beam thickness, width, length $L_{2}$, and material properties as listed in Table 1 remained unchanged from the original

Table 3: Altered parameters of the modified design.

\begin{tabular}{lcc} 
Parameter & Value & Units \\
\hline \hline$L_{1}$ & 57.5 & $\mathrm{~mm}$ \\
$\theta_{1}$ & 29.5 & $\mathrm{deg}$ \\
$\theta_{2}$ & 22 & $\mathrm{deg}$ \\
$d$ & 10.5 & $\mathrm{~mm}$ \\
\hline
\end{tabular}




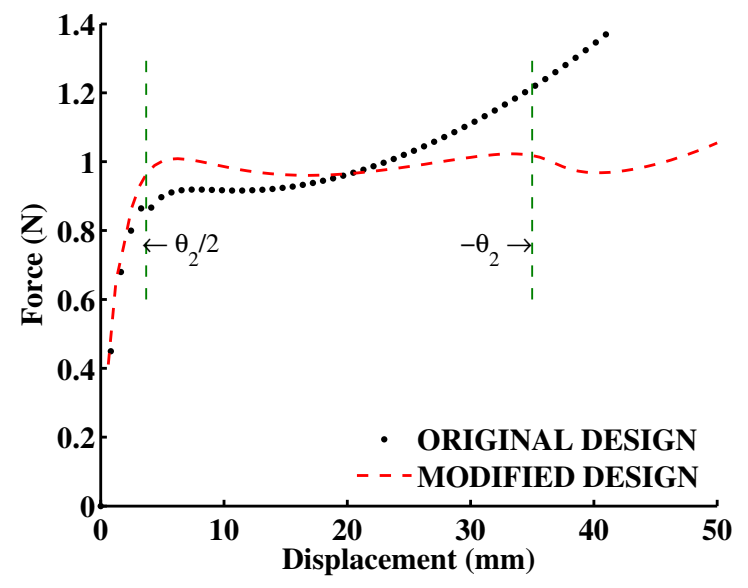

Figure 15: FEA comparison of original mechanism and modified mechanism with optimal beam spacing. The modified optimization range is identified by the vertical dashed lines.

prototype. The model was optimized such that the mechanism would output a constant force of $1 \mathrm{~N}$.

It was found that beams where $\theta_{2}=22^{\circ}$ spaced $10.5 \mathrm{~mm}$ apart were the optimum dimensions for this design. Other dimensions such as $\theta_{1}$ and $L_{1}$ were also altered from the original design and their values are listed in Table 3 . The force-displacement behavior for this mechanism is shown in Figure 15 . The modified design is able to create a larger constant-force displacement than the original design which had the flexures spaced such that the beam ends did not rotate. This modified design was $3 \mathrm{D}$ printed and tested using the same Instron testing setup as discussed in section 4.2 and shown in Figure 8 . The test results in Figure 16 match the FEA results, varying less than $5 \%$.

The modified design offers the advantage of increased constant-force stroke length in the same footprint as the original design. The trade-off of the modified design is that the analysis is more complex and requires a FEA optimization routine whereas the original design is easily modeled using the closed-form equations derived above. The modified design experiences greater stress as one leg will undergo greater strain due to the rotated connecting link, as shown in Figure 14. This design is not as easily adjustable as the original design, as even 200 small design changes require a time consuming optimization routine to determine the ideal parameters for a chosen constant-force.

\section{Applications}

A statically balanced mechanism was constructed as a demonstration of one potential use of the constant-force mechanism. Static balancing is a useful method for reducing actuation efforts in compliant mechanisms [26, 27, 28, 29, 


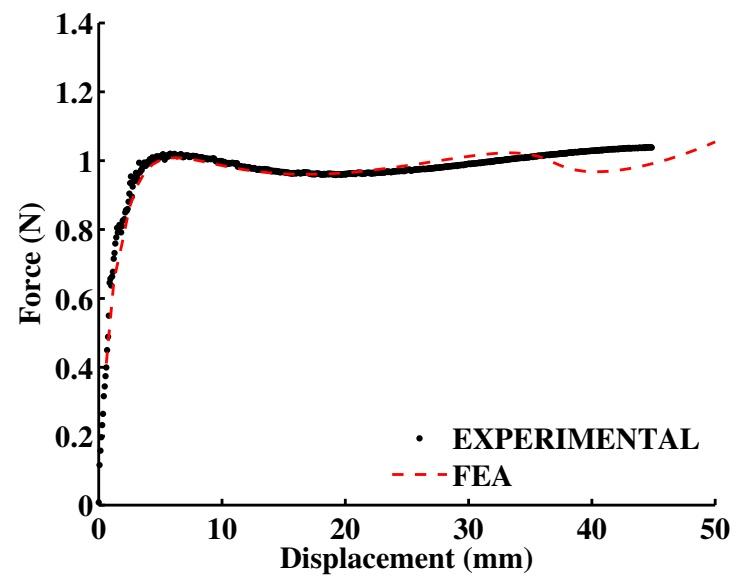

Figure 16: Experimental results of revised design.

30]. It shows promise in surgical and prosthetic applications [31. Research into methods for static balancing is ongoing, including use of the pseudo-rigid-body model [32, 33], numerical methods [34], and the building block approach [35].

The mechanism pictured in Figure 17 is constructed out of PLA and is used to demonstrate the geometry of the system. The device is made of two constant-force mechanisms which are pre-loaded to the middle of their constantforce stroke. This results in a mechanism with significantly reduced resistance to movement of the center slider along the center axis for a given stroke. The mechanism still retains stiffness in all other directions as the center slider is 215 in tension. The resulting effect is like a linear bearing where the slider can move easily along one axis but remains stiff in other directions. By tilting the mechanism one way or another, gravity can cause the center slider to move to one end until it reaches the end of its constant-force stroke and begins to experience a reaction force due to the changing spring constant. This reaction 220 force can cause the slider to oscillate back and forth when excited by an external input.

A static balancing mechanism such as this has the potential to be used in human movement energy generation devices where the slider is a magnet that is moving back and forth through conductive coils. Such energy devices are currently used in handheld charging flashlights where the magnet is moved back and forth in the flashlight housing by shaking it 36. Using a statically balanced mechanism as a linear-motion mechanism has the potential to increase a device's efficiency by reducing energy lost through friction. The mechanism also has the potential to be used in haptic devices [37, or as a linear bearing 230 in harsh environments, such as space, where bearings and sliding parts can encounter problems [38. Although the shown mechanism is 3D printed out of PLA for rapid prototyping purposes, in actual application the statically bal- 


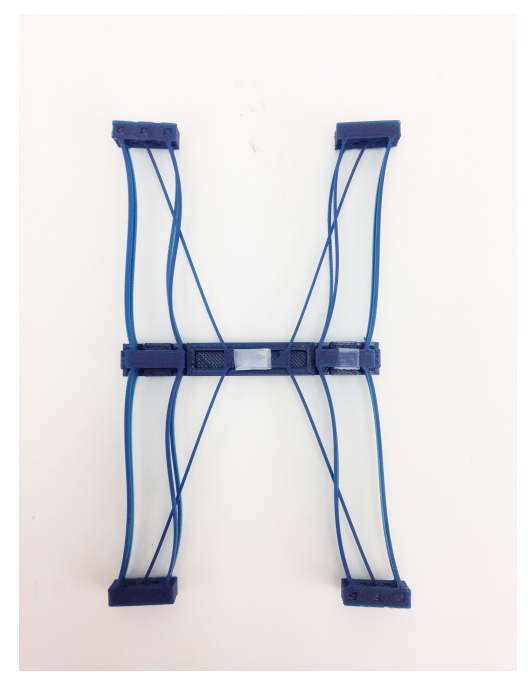

Figure 17: Statically balanced mechanism.

anced mechanism should be fabricated out of steel or another suitable material that avoids the problems of hysteresis and stress relaxation encountered with

\section{Conclusions}

A novel compliant constant-force mechanism based on an initially angled parallel-guiding mechanism has been introduced. A pseudo-rigid-body-model was used in the preliminary design process to identify a constant-force mechanism. The mechanism was shown to be adjustable by keeping the main flexure dimensions constant and by varying the flexure angles. Mechanisms that achieve greater constant-force stroke were designed using FEA optimization routines, with the trade-off of more complex design and mechanisms that are not adjustable. Its potential to be used in statically balanced mechanisms has also 245 been shown. Statically balanced mechanisms consisting of the constant-force mechanism have the potential to be used as compliant linear bearings that do not use ball bearings or sliding joints and has the potential to be used in energy harvesting mechanisms.

\section{Acknowledgments}

250

This work was supported by NASA Office of the Chief Technologists Space Technology Research Fellowships and by the National Science Foundation and the Air Force Office of Scientific Research under NSF Grant EFRI-ODISSEI1240417. 
[1] B. L. Weight, C. A. Mattson, S. P. Magleby, L. L. Howell, Configuration selection, modeling, and preliminary testing in support of constant force electrical connectors, Journal of Electronic Packaging 129 (3) (2007) 236246.

[2] J. C. Meaders, C. A. Mattson, Optimization of near-constant force springs subject to mating uncertainty, Structural and multidisciplinary optimization 41 (1) (2010) 1-15.

[3] H. Porter, Spring-finger diaphragm clutch, US Patent 2,630,897 (Mar. 10 1953).

[4] Z. Li-jun, L. Tao, S. Bao-yu, Optimum design of automobile diaphragm spring clutch, in: Vehicle Power and Propulsion Conference, 2008. VPPC'08. IEEE, IEEE, 2008, pp. 1-4.

[5] R. J. Wilson, Constant force spring powered exercising apparatus, US Patent 4,208,049 (Jun. 17 1980).

[6] L. L. Howell, S. P. Magleby, Substantially constant-force exercise machine, US Patent 7,060,012 (Jun. 13 2006).

[7] C. Boyle, L. L. Howell, S. P. Magleby, M. S. Evans, Dynamic modeling of compliant constant-force compression mechanisms, Mechanism and machine theory 38 (12) (2003) 1469-1487.

[8] C.-C. Lan, J.-H. Wang, Y.-H. Chen, A compliant constant-force mechanism for adaptive robot end-effector operations, in: Robotics and Automation (ICRA), 2010 IEEE International Conference on, IEEE, 2010, pp. 21312136 .

[9] J. Wang, C. Lan, A constant-force compliant gripper for handling objects of various sizes, Journal of Mechanical Design 136 (7).

[10] Y.-H. Chen, C.-C. Lan, An adjustable constant-force mechanism for adaptive end-effector operations, Journal of Mechanical Design 134 (3) (2012) 031005 .

[11] H.-T. Pham, D.-A. Wang, A constant-force bistable mechanism for force regulation and overload protection, Mechanism and Machine Theory 46 (7) (2011) 899-909.

[12] Y.-H. Chen, C.-C. Lan, Design of a constant-force snap-fit mechanism for minimal mating uncertainty, Mechanism and Machine Theory 55 (2012) $34-50$.

[13] Q. T. Aten, S. A. Zirbel, B. D. Jensen, L. L. Howell, A numerical method for position analysis of compliant mechanisms with more degrees of freedom than inputs, Journal of Mechanical Design 133 (6) (2011) 061009. 
[14] H. Wang, J. Zhao, R. Gao, Y. Yang, A novel constant-force scanning probe incorporating mechanical-magnetic coupled structures, Review of Scientific Instruments 82 (7) (2011) 075101.

[15] A. M. Wahl, Mechanical Springs, 2nd Edition, McGraw-Hill, New York, 1963.

[16] R. Nathan, A constant force generation mechanism, Journal of Mechanical Design 107 (4) (1985) 508-512.

[17] J. Jenuwine, A. Midha, Synthesis of single-input and multiple-output port mechanisms with springs for specified energy absorption, Journal of Mechanical design 116 (3) (1994) 937-943.

[18] G. Chen, H. Chang, G. Li, Design of constant-force compliant sarrus mechanism considering stiffness nonlinearity of compliant joints, in: Advances in Reconfigurable Mechanisms and Robots II, Springer, 2016, pp. 107-116.

[19] H. Zhou, H. N. Prakashah, Synthesis of constant torque compliant mechanisms, in: ASME 2015 International Design Engineering Technical Conferences and Computers and Information in Engineering Conference, American Society of Mechanical Engineers, 2015, pp. V05AT08A002V05AT08A002.

[20] R. L. Harne, Z. Wu, K.-W. Wang, Designing and harnessing the metastable states of a modular metastructure for programmable mechanical properties adaptation, Journal of Mechanical Design 138 (2) (2016) 021402.

[21] Z.-W. Yang, C.-C. Lan, An adjustable gravity-balancing mechanism using planar extension and compression springs, Mechanism and Machine Theory 92 (2015) 314-329.

[22] T.-H. Wu, C.-C. Lan, Design and analysis of an elastic mechanism with adjustable zero-to-infinite linear stiffness, in: Advanced Intelligent Mechatronics (AIM), 2015 IEEE International Conference on, IEEE, 2015, pp. 1084-1089.

[23] G. Berselli, R. Vertechy, G. Vassura, V. P. Castelli, Design of a singleacting constant-force actuator based on dielectric elastomers, Journal of Mechanisms and Robotics 1 (3) (2009) 031007.

[24] L. L. Howell, Compliant mechanisms, John Wiley \& Sons, 2001.

[25] A. Midha, S. G. Bapat, A. Mavanthoor, V. Chinta, Analysis of a fixedguided compliant beam with an inflection point using the pseudo-rigid-body model concept, Journal of Mechanisms and Robotics 7 (3) (2015) 031007.

[26] N. Tolou, V. A. Henneken, J. L. Herder, Statically balanced compliant micro mechanisms (sb-mems): Concepts and simulation, in: ASME 2010 International Design Engineering Technical Conferences and Computers and 
[27] J. L. Herder, Design of spring force compensation systems, Mechanism and Machine Theory 33 (12) (1998) $151-161$.

[28] A. Stapel, J. L. Herder, Feasibility study of a fully compliant statically balanced laparoscopic grasper, Vol. 2004, ASME, 2004, pp. 635-643.

[29] E. G. Merriam, L. L. Howell, Non-dimensional approach for static balancing of rotational flexures, Mechanism and Machine Theory 84 (2015) 90-98.

[30] S. R. Deepak, A. N. Hansoge, G. Ananthasuresh, Application of rigid-bodylinkage static balancing techniques to reduce actuation effort in compliant mechanisms, Journal of Mechanisms and Robotics 8 (2) (2016) 021005.

[31] A. Lamers, J. A. G. Sánchez, J. L. Herder, Design of a statically balanced fully compliant grasper, Mechanism and Machine Theory 92 (2015) 230239.

[32] A. Martini, M. Troncossi, M. Carricato, A. Rivola, Static balancing of a parallel kinematics machine with linear-delta architecture: theory, design and numerical investigation, Mechanism and Machine Theory 90 (2015) $128-141$.

[33] S. Perreault, P. Cardou, C. Gosselin, Approximate static balancing of a planar parallel cable-driven mechanism based on four-bar linkages and springs, Mechanism and Machine Theory 79 (2014) 64-79.

[34] C. Kim, D. Ebenstein, Curve decomposition for large deflection analysis of fixed-guided beams with application to statically balanced compliant mechanisms, Journal of Mechanisms and Robotics 4 (4) (2012) 041009.

[35] K. Hoetmer, G. Woo, C. Kim, J. Herder, Negative stiffness building blocks for statically balanced compliant mechanisms: design and testing, Journal

[36] S. Cheng, C. Cepnik, D. P. Arnold, Electrodynamic Vibrational Energy Harvesting, Wiley-VCH Verlag GmbH \& Co. KGaA, 2015, pp. 175-200.

[37] L. C. Leishman, D. J. Ricks, M. B. Colton, Design and evaluation of statically balanced compliant mechanisms for haptic interfaces, in: ASME 2010 Engineers, 2010, pp. 859-866.

[38] R. M. Fowler, L. L. Howell, S. P. Magleby, Compliant space mechanisms: a new frontier for compliant mechanisms, Mechanical Sciences 2 (2) (2011) $205-215$. 